\title{
Impact of the coronavirus disease 2019 (COVID-19) pandemic on nosocomial Clostridioides difficile infection
}

\author{
Manuel Ponce-Alonso MSc ${ }^{1,2}$ (1), Javier Sáez de la Fuente PharmD ${ }^{3}$, Angela Rincón-Carlavilla MD \\ Paloma Moreno-Nunez MD ${ }^{4}$, Laura Martínez-García PharmD ${ }^{1}$, Rosa Escudero-Sánchez MD ${ }^{2,5}$, \\ Rosario Pintor PharmD, $\mathrm{PhD}^{3}$, Sergio García-Fernández PharmD, $\mathrm{PhD}^{1,2}$ and Javier Cobo MD, PhD ${ }^{2,5}$ \\ ${ }^{1}$ Servicio de Microbiología, Hospital Universitario Ramón y Cajal and Instituto Ramón y Cajal de Investigación Sanitaria, Madrid, Spain, ${ }^{2}$ Red Española de \\ Investigación en Patología Infecciosa, Madrid, Spain, ${ }^{3}$ Servicio de Farmacia Hospitalaria, Hospital Universitario Ramón y Cajal and Instituto Ramón y Cajal de \\ Investigación Sanitaria, Madrid, Spain, ${ }^{4}$ Servicio de Medicina Preventiva, Hospital Universitario Ramón y Cajal and Instituto Ramón y Cajal de Investigación \\ Sanitaria, Madrid, Spain and ${ }^{5}$ Servicio de Enfermedades Infecciosas, Hospital Universitario Ramón y Cajal and Instituto Ramón y Cajal de Investigación Sanitaria, \\ Madrid, Spain
}

\begin{abstract}
Objectives: The coronavirus disease 2019 (COVID-19) pandemic has induced a reinforcement of infection control measures in the hospital setting. Here, we assess the impact of the COVID-19 pandemic on the incidence of nosocomial Clostridioides difficile infection (CDI).

Methods: We retrospectively compared the incidence density (cases per 10,000 patient days) of healthcare-facility-associated (HCFA) CDI in a tertiary-care hospital in Madrid, Spain, during the maximum incidence of COVID-19 (March 11 to May 11, 2020) with the same period of the previous year (control period). We also assessed the aggregate in-hospital antibiotic use (ie, defined daily doses [DDD] per 100 occupied bed days $[\mathrm{BD}]$ ) and incidence density (ie, movements per 1,000 patient days) of patient mobility during both periods.

Results: In total, 2,337 patients with reverse transcription-polymerase chain reaction-confirmed COVID-19 were admitted to the hospital during the COVID-19 period. Also, 12 HCFA CDI cases were reported at this time (incidence density, 2.68 per 10,000 patient days), whereas 34 HCFA CDI cases were identified during the control period (incidence density, 8.54 per 10,000 patient days) $(P=.000257)$. Antibiotic consumption was slightly higher during the COVID-19 period (89.73 DDD per $100 \mathrm{BD})$ than during the control period (79.16 DDD per $100 \mathrm{BD})$. The incidence density of patient movements was 587.61 per 1,000 patient days during the control period and was significantly lower during the COVID-19 period (300.86 per 1,000 patient days) $(P<.0001)$.

Conclusions: The observed reduction of $\sim 70 \%$ in the incidence density of HCFA CDI in a context of no reduction in antibiotic use supports the importance of reducing nosocomial transmission by healthcare workers and asymptomatic colonized patients, reinforcing cleaning procedures and reducing patient mobility in the epidemiological control of CDI.
\end{abstract}

(Received 30 June 2020; accepted 24 August 2020; electronically published 8 September 2020)

Clostridioides difficile is the leading cause of nosocomial infectious diarrhea and one of the most prevalent nosocomial pathogens. ${ }^{1,2}$ The key elements that determine its incidence are exposure to $C$. difficile spores and the administration of antibiotics. ${ }^{3}$ Controversy exists over the utility of various infection control measures, given that most interventions have shown very low levels of evidence, ${ }^{4}$ whereas bundle-based programs that include antibiotic restriction are almost always effective. ${ }^{4,5}$

The COVID-19 pandemic in Spain has been particularly intense, with $>6,000$ cases per million inhabitants and exceeding 28,000 deaths $^{6}$; it was even more serious in the capital Madrid. Our hospital suddenly became a monographic COVID-19 hospital, with a peak in the pandemic on March 30, 2020, when $86.91 \%$ of admitted patients $(983$ of 1,131$)$ were diagnosed with

Author for correspondence: Manuel Ponce-Alonso, E-mail: lugonauta@gmail.com Cite this article: Ponce-Alonso M, et al. (2021). Impact of the coronavirus disease 2019 (COVID-19) pandemic on nosocomial Clostridioides difficile infection. Infection Control \& Hospital Epidemiology, 42: 406-410, https://doi.org/10.1017/ice.2020.454
COVID-19. This situation in which almost all patients remained isolated and all healthcare workers wore personal protective equipment (PPE) constituted a type of "natural experiment" for the study of $C$. difficile epidemiology in hospitals. The objective of this study was to assess the impact of the COVID-19 pandemic on the incidence of CDI and to analyze the factors that could have influenced the incidence.

\section{Methods}

We compared the incidence density (cases per 10,000 patient days) of nosocomial CDI in a tertiary-care teaching hospital in Madrid over 2 periods: (1) the peak incidence of COVID-19 at our hospital (COVID-19 period: March 11, 2020, to May 11, 2020) and (2) the same period of the previous year (control period: March 11, 2019, to May 11, 2019). We used the standard epidemiological classification of $\mathrm{CDI}^{7}{ }^{7}$ only considering hospital-onset healthcare facility-associated (HO-HCFA) and community-onset healthcare facility-associated (CO-HCFA) infections as nosocomial, and 
ruling out community, indeterminate, and recurrence cases for the incidence density calculation. We also calculated the incidence density for all types of CDI during the interval between the 2 periods to better describe the time trend of cases. For the COVID-19 period, we reviewed whether the hospitalized patients who were screened for CDI also presented a diagnosis of COVID-19, with microbiological confirmation by reverse transcription-polymerase chain reaction (RT-PCR). We used the computerized registry of the microbiology department to obtain all data regarding CDI and COVID-19 tests, and we gathered hospital admission data regarding hospital stays. The algorithm employed for the microbiological diagnosis of CDI was the same throughout the study period, which included sequential qualitative detection of glutamate dehydrogenase (C. DIFF QUIK CHEK, TechLab, Blacksburg, VA) and A and B toxins (TOX A/B QUIK CHEK, TechLab) from $C$. difficile. We assessed discrepancies by detecting the C. difficile toxin B gene by RT-PCR (BD MAX Cdiff, Becton Dickinson, Franklin Lakes, NJ).

We assessed patient mobility by determining all of the patients' administrative location changes during both periods using the hospital information systems service. These changes included transfers to the operating room, change in room or change to intensive care units, as well as transfers to perform additional tests (eg, radiological examinations, endoscopies, or other procedures). We then calculated the "incidence density" of the patients' movements by dividing the sum of the location changes by the hospital stays in both periods.

For the same study period, we extracted aggregate in-hospital antibiotic use data from the computerized hospital administration records and an automated medication-dispensing system. Data on antibiotic use are expressed as defined daily doses per $100 \mathrm{occu}-$ pied bed days (DDD per $100 \mathrm{BD}$ ) and the percentage of change between study periods, according to the criteria of the World Health Organization Collaborating Centre for Drug Statistics Methodology. We also extracted data regarding time of exposure to antibiotics, expressed as days of therapy (DOT) per 100 patient days. For this study, we considered only the consumption of antibiotics from Anatomical Therapeutic Chemical group J01. Finally, as a subrogate measurement of antibiotic exposure, the proportion of admitted patients who suffered from at least 1 episode of microbiologically confirmed nonviral infection was calculated for both periods.

We compared the categorical data using the $\chi^{2}$ test and the Student $t$ test to compare the continuous variables when a normal distribution could be assumed or the Mann-Whitney U test otherwise. We assessed the normality of the continuous variables using the Shapiro-Wilk test.

\section{Results}

In total, 2,337 patients with RT-PCR-confirmed COVID-19 and 283 with suspected COVID-19 were admitted to the hospital during the COVID-19 period. There were 44,831 hospital stays during this time, whereas during the same period of 2019 there were 39,795 stays. The mean age of the admitted patients was $66.74 \pm 17.65$ years for the COVID-19 period and $64.41 \pm 21.23$ years for the control period $(P<.0001)$.

\section{Clostridioides difficile infection cases}

The total requests for $C$. difficile detection in the hospitalized patients were similar between the COVID-19 period $(n=209)$ and the control period $(n=203)$, with a $9.8 \%$ reduction in the rate of requests during the COVID-19 period (4.6 per 1,000 hospital stays vs 5.1 per 1,000 hospital stays). Also, 12 HCFA CDI cases were identified during the COVID-19 period (10 corresponding to HO-HCFA and 2 to CO-HCFA), which resulted in an HCFA CDI incidence density of 2.68 per 10,000 patient days. In the control period, we identified $34 \mathrm{HCFA}$ CDI cases (21 corresponding to HO-HCFA and 13 to CO-HCFA), which resulted in an HCFA CDI incidence density of 8.54 per 10,000 patient days. Thus, the HCFA CDI incidence density was $\sim 3$ times lower for the COVID-19 period than for the non-COVID-19 period (incidence rate ratio, $0.31 ; 95 \% \mathrm{CI}, 0.16-0.61 ; P=.000257)$. Figure 1 shows the evolution of CDI cases and the incidence density of HCFA CDI during the 2 study periods and the 10-month interval between them.

Among the 209 hospitalized patients screened for CDI during the COVID-19 period, a lower proportion of CDI was observed in those with RT-PCR-confirmed COVID-19 (5 of 106; 4.7\%) compared with that observed in patients without a microbiological diagnosis of COVID-19 (8 of 103; 7.8\%). However, these differences were not statistically significant $(P=.36)$.

\section{Patient mobility}

Patient mobility was drastically reduced during the COVID-19 period, with 4,858 bed movements (2,274 involving a nursing unit change), whereas 7,338 bed movements $(1,877$ involving a nursing unit change) were observed during the control period. The number of surgical interventions was reduced to 668 during the COVID-19 period, contrasting with the 2,227 surgeries observed during the control period. The total numbers of diagnostic or care procedures that involved patient movement were 7,963 for the COVID-19 period and 13,819 for the control period. The incidence density of movements was 300.86 per 1,000 patient days for the COVID-19 period and 587.61 per 1,000 patient days for the control period $(P<.0001)$.

\section{Antibiotic consumption}

The consumption of antibiotics measured by DDD per $100 \mathrm{BD}$ was higher during the COVID-19 period (89.73 DDD per $100 \mathrm{BD}$ ) than during the control period (79.16 DDD per $100 \mathrm{BD})$, remaining stable during the months between the 2 periods (77.50 \pm 1.64 DDD per $100 \mathrm{BD})$. The qualitative analysis showed a dramatic increase during the COVID-19 period in the use of third-generation cephalosporins (6.34 DDD per $100 \mathrm{BD}$ for the control period vs 19.39 DDD per $100 \mathrm{BD}$ for the COVID-19 period) and macrolides (5.76 DDD per $100 \mathrm{BD}$ vs $25.49 \mathrm{DDD}$ per $100 \mathrm{BD}$, respectively). We observed the same trend in terms of time of exposure to both third-generation cephalosporins (6.64 DOT per 100 patient days for the control period vs 20.98 DOT per 100 patient days for the COVID-19 period) and macrolides (3.97 DOT per 100 patient days vs 15.97 DOT per 100 patient days, respectively). Conversely, we observed a remarkable reduction in the consumption of quinolones (8.68 DDD per $100 \mathrm{BD}$ for the control period vs $4.61 \mathrm{DDD}$ per $100 \mathrm{BD}$ for the COVID-19 period) and inhibitor-penicillin combinations (25.7 DDD per $100 \mathrm{BD}$ vs $16.60 \mathrm{DDD}$ per 100 $\mathrm{BD}$, respectively). Treatment duration was also reduced for quinolones (8.14 DOT per 100 patient days for the control period vs 3.98 DOT per 100 patient days for the COVID-19 period) and inhibitor-penicillin combinations (27.94 DOT per 100 patient days vs 17.95 DOT per 100 patient days, respectively). There were no relevant differences in the use or in treatment duration of carbapenems between the 2 periods (7.42 DDD per $100 \mathrm{BD}$ for the 


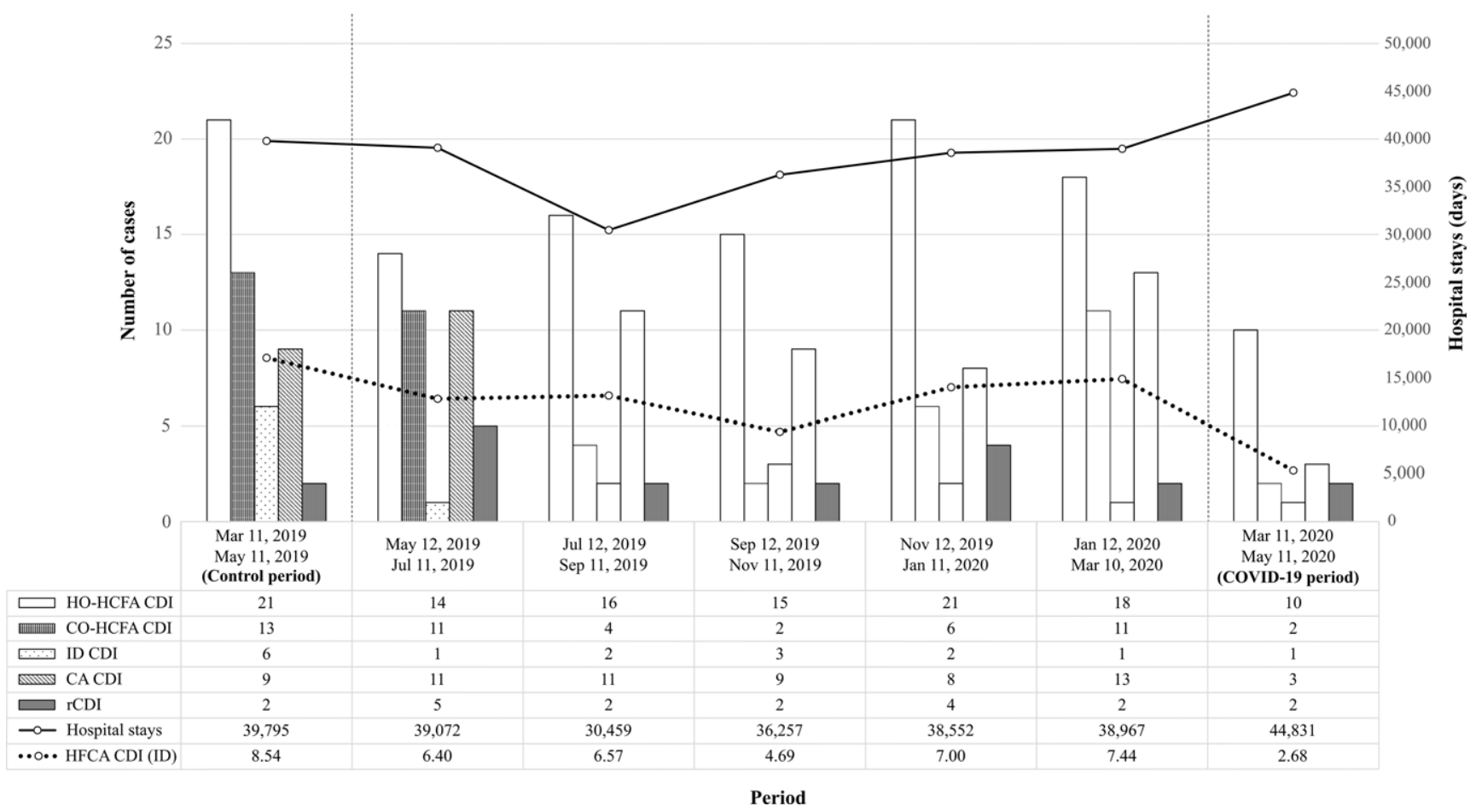

Fig. 1. Evolution of $C$. difficile infection (CDI) over time, from control period (left) to COVID-19 period (right). The bar chart shows the total CDI case count, grouped by epidemiological definition. The solid line represents total hospital stays during each period (in days), which were used to calculate the incidence density of nosocomial CDI cases (dashed line). Note. HO-HCFA CDI, hospital-onset healthcare facility-associated C. difficile infection; CO-HCFA CDI, community-onset healthcare facility-associated $C$. difficile infection; ID CDI, indeterminate-onset $C$. difficile infection; CA CDI, community-acquired $C$. difficile infection; rCDI, recurrent $C$. difficile infection; HCFA CDI (ID), incidence density of healthcare facility-associated $C$. difficile infection.

control period vs 6.34 DDD per $100 \mathrm{BD}$ for the COVID-19 period; 8.06 DOT per 100 patient days vs 6.93 DOT per 100 patient days, respectively). Finally, as an indirect measurement of antibiotic exposure, the proportion of admitted patients who suffered from at least 1 episode of microbiologically confirmed nonviral infection was higher during the control period (15.5\%; 984 of 6,365 patients) than during the COVID-19 period (14\%; 783 of 5,600 patients).

\section{Infection control measures}

An infection prevention bundle was implemented during the COVID-19 period (Table 1). All healthcare workers wore personal protective equipment (PPE) when caring for patients with COVID-19 and wore nonwaterproof masks, gloves, and gowns when treating patients without COVID-19. Environmental cleaning by trained cleaning staff was reinforced, and visits were prohibited (except in exceptional situations). Patients with COVID-19 were grouped in wards, with rooms intended for a maximum of 2 patients; however, a third bed had to be included in some cases. Patients with CDI were isolated in a single room with contact precautions during both the COVID-19 and control periods. Moreover, the same cleaning products were employed for both periods.

\section{Discussion}

Our results show a remarkable reduction in the incidence density of nosocomial CDI during the period with the maximum incidence of COVID-19 compared with the same period the previous year. The number of CDI cases remained stable in the previous months; thus, such a decrease during the COVID-19 period cannot be explained by the previous trend. Moreover, the number of CDI test requests for the hospitalized patients from both periods was similar; therefore, there is no reason to envision a reduction in the clinical suspicion of CDI in patients with diarrhea (despite the frequent use of lopinavir-ritonavir as COVID-19 therapy).

Based on our findings, the consumption of antibiotics does not appear to explain the decrease in CDI. Although the use of quinolones was reduced, the overall consumption of antibiotics increased during the COVID-19 period. This observation contrasts with the lower proportion of admitted patients who suffered from at least 1 episode of microbiologically confirmed nonviral infection during the COVID-19 period (14\%), compared to the control period (15.5\%). The increment in antibiotic consumption could be explained by the fact that, for a time, the institutional protocol for the treatment of patients with COVID-19 contemplated the optional use of empiric ceftriaxone, and many doctors added it as standard regimen from the emergency room. In fact, a recent systematic review ${ }^{8}$ pointed out that $71.9 \%$ of patients with COVID-19 received antibiotics despite the low rate of bacterial infection observed in those patients (6.9\%). This finding could explain the noticeable increase in both the use and time of exposure to third-generation cephalosporins observed in our study, a subgroup of antimicrobial agents associated with a higher risk for developing $\mathrm{CDI}^{9}$.

Due to the exceptional epidemiological situation during the COVID-19 period, our institution introduced an extraordinary reinforcement of all infection control measures, including patient isolation, universal PPE, limited patient visits and movement, and reinforcement of cleaning regimens, all of which have indirectly limited the nosocomial spread of $C$. difficile. During this period, we observed an almost $70 \%$ reduction in the incidence density of nosocomial CDI. We postulate that this observation confirms 
Table 1. Implemented Bundle to Prevent the Spread of SARS-CoV-2 in our Hospital During the COVID-19 Pandemic

\begin{tabular}{|c|c|}
\hline Bundle Element & Description \\
\hline \multirow[t]{2}{*}{ Personal protective equipment (PPE) } & $\begin{array}{l}\text { All healthcare workers wore PPE (masks, gloves, goggles, caps and waterproof gowns) to care for } \\
\text { patients with COVID-19 }\end{array}$ \\
\hline & Training healthcare workers on the proper use of PPE \\
\hline Patient location & Individual rooms/grouping of cases \\
\hline \multirow[t]{5}{*}{ Isolation precautions } & Design and diffusion of specific posters \\
\hline & Isolation precautions for almost all hospitalized patients \\
\hline & Isolation of a confirmed/suspected patient in $<24 \mathrm{~h}$ through an ad hoc alert system \\
\hline & Isolation measures placed very visibly in the patient's medical chart \\
\hline & Increased availability of infection control staff for information and incident resolution \\
\hline \multirow[t]{10}{*}{$\begin{array}{l}\text { Patient environment (rooms, common areas and } \\
\text { transit areas) }\end{array}$} & $\begin{array}{l}\text { Reinforcement of the daily communication between infection control staff, cleaning staff and } \\
\text { management team }\end{array}$ \\
\hline & Reinforcement of the cleaning staff in all hospitalization areas \\
\hline & $\begin{array}{l}\text { Operation check of chlorinated product dispensing pumps to ensure adequate concentrations }(3,000 \\
\text { ppm) }\end{array}$ \\
\hline & Training reinforcement for cleaning staff \\
\hline & $\begin{array}{l}\text { Adaptation of the usual cleaning procedures and creation of specific protocols for SARS-CoV- } 2 \\
\text { eradication }\end{array}$ \\
\hline & $\begin{array}{l}\text { Design of a poster with the most relevant points of each cleaning procedure and placement in visible } \\
\text { areas }\end{array}$ \\
\hline & Acquisition of additional cleaning material to prevent its reuse \\
\hline & Daily audit of scheduled cleaning by the preventive medicine department \\
\hline & Schedule of special cleaning adapted to the needs detected in the audits \\
\hline & $\begin{array}{l}\text { Reinforcement of the cleaning of common areas with sodium hypochlorite sprays by the military } \\
\text { emergency unit }\end{array}$ \\
\hline \multirow[t]{3}{*}{ Sanitary material } & Training healthcare workers on disinfection procedures of sanitary material \\
\hline & Increased availability of disinfectant products \\
\hline & Audit of the proper use of cleaning products and disinfection procedures \\
\hline \multirow[t]{2}{*}{ Health worker environment } & $\begin{array}{l}\text { Training healthcare workers on the need to disinfect counters, computer equipment, and personal } \\
\text { items after use }\end{array}$ \\
\hline & Cleaning of the common and rest areas of health personnel after each work shift \\
\hline Patient movements & Transfers limited to what is strictly essential (diagnostic or therapeutic procedures) \\
\hline Visits & Extension of the prohibition of visits and companions to almost all situations \\
\hline Waste management & Reduction of waste movement by installing class III waste containers inside all rooms \\
\hline Hand hygiene & Training reinforcement on hand hygiene practices \\
\hline
\end{tabular}

the importance of strategies aimed at reducing nosocomial transmission of C. difficile. Notably, not only the reinforcement of infection control measures but also the exceptionally dramatic situation during the COVID-19 period could have contributed to an increase in adherence to those measures by healthcare workers, as has already been pointed out. ${ }^{10,11}$

The extension of containment measures to all of our hospitalized patients during the COVID-19 pandemic could have also limited transmission from asymptomatic patients, who represent an important source of transmission, ${ }^{12,13}$ despite this group transmitting less effectively. ${ }^{14}$ In addition, the suppression of consultations and surgical procedures in the hospital has meant fewer opportunities for introducing $C$. difficile into the hospital from the community.

Our results contrast with those of a recent study that found no benefits on the incidence of CDI from improving hospital cleaning procedures..$^{15}$ Closing hospitals and transferring patients to individual rooms located in new facilities has not conclusively been associated with a reduction in CDI rates ${ }^{16,17}$; however, our results support previous studies that linked the mobility of patients to common areas with increased risk of developing $\mathrm{CDI}^{9}$ and the potential risk of transmission by the hands of healthcare workers. ${ }^{18,19}$ Notably, in both cases, transmission was not associated only with direct contact with symptomatic patients ${ }^{20}$ and multidisciplinary measures were necessary to limit the spread of $C$. difficile.

The retrospective nature of our study precludes us from controlling for numerous factors and from measuring certain variables in detail, such as the degree of compliance with cleaning and the previous state of $C$. difficile colonization of patients, which might have been lower than usual upon admission. We also cannot rule out the possibility that our COVID-19 population was mostly composed of previously healthy patients, although our data 
showed that patients admitted during the COVID-19 period were significantly older than those admitted during the control period. Another limitation of our study lies in the effect of the COVID-19 pandemic on outpatient care, as recently highlighted in other more serious diseases. ${ }^{21}$ This factor could explain part of the reduction observed in nosocomial CDI cases by reducing opportunities for CO-HCFA diagnosis and requests for care due to the COVID19 epidemic. Nevertheless, even considering only HO-HCFA cases, the observed reduction was $\sim 50 \%$.

Despite the aforementioned limitations, our observation of a dramatic decrease in CDI in a context of no reduction in the use of antibiotics supports the importance of reducing the nosocomial transmission by healthcare workers or asymptomatically colonized patients, reinforcing cleaning procedure and reducing hospital mobility of patients in the epidemiological control of CDI.

Acknowledgments. The authors thank Gerardo Gómez Montero, Department of Information Systems of Hospital Ramón y Cajal, for his contributions in the collection of patient movement records.

Financial support. This work was supported by the Instituto de Salud Carlos III and REIPI (grant no. 0D16/0016/0011), cofinanced by the European Development Regional Fund "A Way to Achieve Europe" (ERDF). M. Ponce-Alonso was supported by a research contract from Instituto de Salud Carlos III, Spain (Rio Hortega program, grant no. CM19/00069). L. Martínez-García was supported by a research contract from Instituto de Salud Carlos III, Spain (Rio Hortega program, grant no. CM19/00136).

Conflicts of interest. All authors report no conflicts of interest relevant to this article.

\section{References}

1. Lessa FC, Mu Y, Bamberg WM, et al. Burden of Clostridium difficile infection in the United States. N Engl J Med 2015;372:825-34.

2. Davies KA, Longshaw CM, Davis GL, et al. Underdiagnosis of Clostridium difficile across Europe: the European, multicentre, prospective, biannual, point-prevalence study of Clostridium difficile infection in hospitalised patients with diarrhoea (EUCLID). Lancet Infect Dis 2014;14:1208-1219.

3. Longo DL, Leffler DA, Lamont JT. Clostridium difficile infection. $N$ Engl J Med 2015;372:1539-1548.

4. Tschudin-Sutter S, Kuijper EJ, Durovic A, et al. Guidance document for prevention of Clostridium difficile infection in acute healthcare settings. Clin Microbiol Infect 2018;24:1051-1054.

5. Barker AK, Ngam C, Musuuza JS, Vaughn VM, Safdar N. Reducing Clostridium difficile in the inpatient setting: a systematic review of the adherence to and effectiveness of $C$. difficile prevention bundles. Infect Control Hosp Epidemiol 2017;38:639-650.
6. Spain coronavirus: cases and deaths. Worldometer website. https://www. worldometers.info/coronavirus/country/spain/. Accessed June 15, 2020.

7. Surawicz CM, Brandt LJ, Binion DG, et al. Guidelines for diagnosis, treatment, and prevention of Clostridium difficile infections. Am J Gastroenterol 2013;108:478-98.

8. Langford BJ, So M, Raybardhan S, et al. Bacterial co-infection and secondary infection in patients with COVID-19: a living rapid review and metaanalysis. Clin Microbiol Infect 2020. doi: 10.1016/j.cmi.2020.07.016.

9. Murray SG, Yim JWL, Croci R, et al. Using spatial and temporal mapping to identify nosocomial disease transmission of Clostridium difficile. JAMA Intern Med 2017;177:1863-1865.

10. Israel S, Harpaz K, Radvogin E, et al. Dramatically improved hand hygiene performance rates at time of coronavirus pandemic. Clin Microbiol Infect 2020. doi: $10.1016 /$ j.cmi.2020.06.002.

11. Houghton C, Meskell P, Delaney H, et al. Barriers and facilitators to healthcare workers' adherence with infection prevention and control (IPC) guidelines for respiratory infectious diseases: a rapid qualitative evidence synthesis. Cochrane Database Syst Rev 2020;4(4):CD013582.

12. Longtin Y, Paquet-Bolduc B, Gilca R, et al. Effect of detecting and isolating Clostridium difficile carriers at hospital admission on the incidence of C. difficile infections. JAMA Intern Med 2016;176:796-717.

13. Crobach MJT, Vernon JJ, Loo VG, et al. Understanding Clostridium difficile colonization. Clin Microbiol Rev 2018;31:1094-1129.

14. Durham DP, Olsen MA, Dubberke ER, Galvani AP, Townsend JP. Quantifying transmission of Clostridium difficile within and outside healthcare settings. Emerging Infect Dis 2016;22:608-616.

15. Mitchell BG, Hall L, White N, et al. An environmental cleaning bundle and health-care-associated infections in hospitals (REACH): a multicentre, randomised trial. Lancet Infect Dis 2019;19:410-418.

16. Heddema ER, van Benthem BHB. Decline in incidence of Clostridium difficile infection after relocation to a new hospital building with single rooms. J Hosp Infect 2011;79:93-94.

17. McDonald EG, Dendukuri N, Frenette C, Lee TC. Time-series analysis of health care-associated infections in a new hospital with all private rooms. JAMA Intern Med 2019. doi: 10.1001/jamainternmed.2019.2798.

18. Jullian-Desayes I, Landelle C, Mallaret MR, Brun-Buisson C, Barbut F. Clostridium difficile contamination of health care workers' hands and its potential contribution to the spread of infection: review of the literature. Am J Infect Control 2017;45:51-58.

19. Landelle C, Verachten $M$, Legrand $P$, et al. Contamination of healthcare workers' hands with Clostridium difficile spores after caring for patients with $C$. difficile infection. Infect Control Hosp Epidemiol 2014;35:10-5.

20. García-Fernández S, Frentrup M, Steglich $M$, et al. Whole-genome sequencing reveals nosocomial Clostridioides difficile transmission and a previously unsuspected epidemic scenario. Sci Rep 2019;9:6959.

21. Tam CCF, Cheung KS, Lam S, et al. Impact of coronavirus disease 2019 (COVID-19) outbreak on outcome of myocardial infarction in Hong Kong, China. Catheter Cardiovasc Interv 2020;91:157-154. 